\title{
Reply to Azithromycin: Short Course with Long Duration
}

\author{
Stephanie Royer, MD ${ }^{1,2,3 *}$, Hallie C. Prescott MD ${ }^{1,4}$ \\ 'Department of Internal Medicine, University of Michigan, Ann Arbor, Michigan; ${ }^{2}$ Division of Hospital Medicine, Cincinnati Children's Hospital \\ Medical Center, Cincinnati, Ohio; ${ }^{3}$ Department of Internal Medicine, University of Cincinnati, Cincinnati, Ohio; ${ }^{4}$ A Center for Clinical Management \\ Research, VA Ann Arbor Healthcare System, Ann Arbor, Michigan.
}

W e appreciate the interest in our review of antibiotic duration in hospitalized patients. Drs. Sikkens and van Agtmael comment that drug pharmacokinetics can alter true treatment duration. ${ }^{1,2}$ Specifically, azithromycin has a long half-life in tissues. ${ }^{3}$ We did not consider pharmacokinetics in our prespecified protocol for study inclusion, nor require that studies compare the same drug between treatment groups. This is consistent with a systematic review of antibiotic duration in community-acquired pneumonia, which included 3 of the 4 studies comparing shortcourse azithromycin to a longer course of another antibiotic. ${ }^{4}$ Similarly, in a recent pilot study of antibiotic duration in bloodstream infections, only treatment duration was prespecified. ${ }^{5}$ We agree that the differing pharmacokinetics between drugs is a limitation to our findings.

To assess whether the inclusion of studies using short-course azithromycin biased our conclusions, we performed an additional meta-analysis for clinical efficacy excluding the 4 studies that compared azithromycin with another drug. This subgroup

*Address for correspondence: Stephanie Royer, MD, 3333 Burnet Avenue, MLC 3024, Cincinnati, OH, 45229; Telephone: (513) 636-5148; Fax: (513) 8039245; E-mail: Stephanie.Royer@cchmc.org

Received: March 6, 2018; Accepted: March 11, 2018

() 2018 Society of Hospital Medicine DOI 10.12788/jhm.2983 included 9 trials comprising 1270 patients. The overall risk difference was $0.3 \%(95 \% \mathrm{Cl}-2.7 \%, 3.3 \%)$, consistent with the primary findings that short-course antibiotic treatment is non-inferior to long-course antibiotic treatment. None of these 4 studies examined mortality; thus, the meta-analyses for shortterm and long-term mortality are unaffected.

Disclosures: Dr. Royer holds stock in Pfizer. The authors have no other potential financial conflicts of interest to report.

Funding: This work was supported by K08 GM115859 [HCP]. This manuscript does not necessarily represent the position or policy of the US government or the Department of Veterans Affairs.

\section{References}

1. Sikkens JJ, van Agtmael MA. Azithromycin: short course with long duration. J Hosp Med. 2018;13(7):582.

2. Royer S, DeMerle KM, Dickson RP, Prescott HC. Shorter versus longer courses of antibiotics for infection in hospitalized patients: a systematic review and meta-analysis. J Hosp Med. 2018;13(5):336-342. doi: 10.12788/jhm.2905.

3. Di Paolo A, Barbara C, Chella A, Angeletti CA, Del Tacca M. Pharmacokinetics of azithromycin in lung tissue, bronchial washing, and plasma in patients given multiple oral doses of 500 and 1000 mg daily. Pharmacol Res. 2002;46(6):545-550. doi: 10.1016/S1043661802002384.

4. Li JZ, Winston LG, Moore DH, Bent S. Efficacy of short-course antibiotic regimens for community-acquired pneumonia: a meta-analysis. Am J Med. 2007;120(9):783-790.

5. Daneman N, Rishu AH, Pinto R, et al. 7 versus 14 days of antibiotic treatment for critically ill patients with bloodstream infection: a pilot randomized clinical trial. Trials. 2018;19(1):111. 